\title{
Primary Care Physician Perspective on Management of Patients With Depression
}

\author{
DANIEL E. FORD, MD, MPH
}

\begin{abstract}
OBJECTIVE: To discuss the relationship between the primary care physician and other health care professionals and best practice strategies in the management of depression.

SUMMARY: Useful chronic clinical models can transform a visit between a patient with depression and the primary care provider from an unfocused encounter to one that is anticipated, planned, and productive. With the proper decision and organizational support to manage their patients with depression, primary care providers will have the increased confidence to treat these patients and have more knowledge about the treatment plan, whether that is a referral or continuation of treatment in the provider's office.
\end{abstract}

CONCLUSION: The primary care provider must recognize that he or she cannot work alone. In addition to decision support and clinical information systems, there needs to be a delivery system that supports a depression care manager and identifies the patient as the key player in the system. Participation and cooperation by the entire health care system will determine the success of such depression care models.

KEYWORDS: Depression, Primary care, Chronic disease, Disease management, Delivery of care

J Manag Care Pharm. 2006;12(2)(suppl):S10-S13
$\mathrm{D}$ epression has multiple medical, economic, and social consequences (Table 1). While patients may be concerned about their personal suffering and interpersonal relationships at home and work, primary care physicians (PCPs) are often concerned about patient nonadherence to therapy and obtaining better outcomes for their patients. In addition, because increasing evidence indicates that depression is an independent risk factor for cardiovascular disease and diabetes, mental illness cannot be treated successfully without also taking such medical issues into consideration. Moreover, patients with depression frequently overuse medical services ${ }^{1}$ and markedly drive up primary health care costs. ${ }^{2}$ One health maintenance organization (HMO) calculated the average per-patient cost for enrollees with either condition at $\$ 2,390$ for a 6-month period, compared with $\$ 1,400$ for those without the disorders. This combined overutilization of medical services and nonadherence to prescribed care leads to an inefficient process in which both the patients and providers suffer.

Despite improvement in detection and diagnosis of depression, an estimated $10 \%$ of patients who meet criteria for depression in a primary care setting are treated only with a benzodiazepine. ${ }^{3}$ In addition, psychosocial interventions are rarely being utilized, and antidepressants, when used, are not being appropriately dosed or prescribed for an adequate length of time. Approximately one third of patients stop taking antidepressants within the first 30 days, and rarely do they stay on the antidepressants during the continuation and maintenance phases. ${ }^{4}$ Moreover, while referral to a mental health specialist may be one option for getting patients the right treatment, it is estimated that only $50 \%$ of those referred actually have more than one visit. Most patients will never engage in the mental health system and instead return to the PCP at a later time without any improvement in symptoms.

\section{Comparison of Depression and Chronic Medical Diseases}

Treatment strategies for depression should be similar to those for any other chronic disease. PCPs should be educated on the chronic nature and severity of the depressive illness, and it should be

\section{TABLE 1) Consequences of Depression}

DANIEL E. FORD, MD, MPH, is a professor of medicine and psychiatry and vice dean, clinical investigation, Johns Hopkins University School of Medicine, and professor of epidemiology and health policy and management, JHU Bloomberg School of Public Health, Baltimore, Maryland.

AUTHOR CORRESPONDENCE: Daniel E. Ford, MD, MPH, Professor and Vice Dean, Johns Hopkins University School of Medicine, Suite 2-500 (Room 2-518), 2024 East Monument St., Baltimore, MD 21287-0007. Tel: (410) 614-0986;

Fax: (410)614-0588; E-mail: dford@jhmi.edu

Copyright $\odot$ 2006, Academy of Managed Care Pharmacy. All rights reserved.
- Personal and family suffering

- Suicide

- Functional impairment at work and home

- Underemployment and low educational achievement

- Substance abuse and tobacco smoking

- Nonadherence to medical therapy

- Myocardial infarction, stroke, and probably diabetes

- Overutilization of medical services 
stressed that appropriate treatment of depression is necessary to prevent relapses and/or recurrent episodes. The prevalence of depression is similar to other chronic medical conditions such as asthma and diabetes, and there is a close relationship between comorbidities for depression and such diseases. In a survey of PCPs conducted by the Robert Wood Johnson Depression in Primary Care initiative, approximately $80 \%$ of providers indicated that they should be able to manage at least $75 \%$ of their patients with depression. ${ }^{5}$ Of note, this figure reflected similar opinions about the provider's perceived role in diabetes care. In general, most PCPs expect to provide continual care for patients with chronic disease, including depression.

In addition to recognizing their role in the chronic management of patients with depression, PCPs have struggled to find appropriate outcome measures for the disorder. While the outcome measures are fairly clear with diseases such as diabetes (i.e., $\mathrm{HbAlc}$ ) and hypertension (i.e., blood pressure), they are less certain of monitoring parameters for depression. Instruments like the Patient Health Questionnaire (PHQ) can greatly aid in convincing PCPs that depression is an illness that can be tracked over time with observed gradual improvements in outcome measures. ${ }^{6}$

Depression can be distinguished from other chronic conditions, from the perspective of PCPs, in that there is still a significant stigma associated with the illness, and patients are less accepting of evidence-based treatment. One report suggests that the difference between patients with depression seen in primary care settings and those in mental health settings is the primary care patients' lack of acceptance of evidence-based care (e.g., the use of antidepressants) and not the severity of illness or presence of medical comorbidities. ${ }^{7}$ Approximately 90\% of patients being seen by a mental health specialist will agree to take an antidepressant, while only about $50 \%$ of patients in a primary care setting will readily accept the treatment plan. In addition, some of the difference can be attributed to the insurance benefit. Many patients with depression may already have a financial burden (e.g., loss of employment and insurance or no insurance) or may prefer counseling over medications; unfortunately, PCPs may not be able to provide many answers when patients inquire about the financial aspects of their care.

Another difference between depression and chronic medical conditions for PCPs is that they may feel less confident in their skills to fully manage patients with depression. ${ }^{8}$ PCPs report that they are generally confident in diagnosing depression and managing one antidepressant. The situation becomes more complicated when they have to manage more than one anti-depressant. Only 16\% of PCPs report being very skilled in managing 2 antidepressants, and only $50 \%$ report being very skilled in describing how care would be given in specialty mental health settings. Therefore, it is not hard to speculate that both PCPs and patients may not be aware of the entire referral process.

PCPs are also frustrated by their inability to obtain high-quality specialty mental health care for their patients (Figure 1). In a study

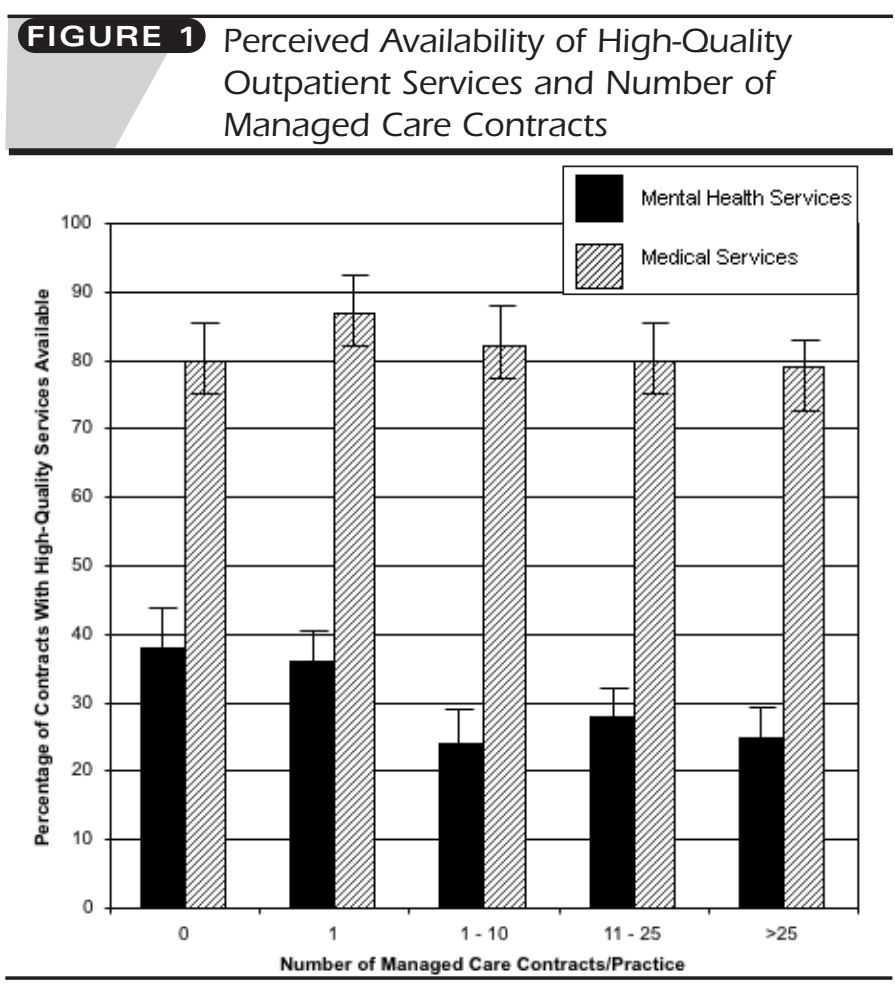

From: Van Voorhees BW et al. Gen Hosp Psych. (2003; 25:149-57.

involving a national sample of PCPs, the availability of specialty medical care was reported for mental health and medical care. ${ }^{9}$ Providers reported that, while $80 \%$ of the time they can obtain high-quality specialty medical care, they can do so only about $20 \%$ of the time for mental health care. This pattern does not appear to be influenced by the number of managed care contracts for specialty medical care; however, there is a distinct downward trend for mental health care as the number of contracts increase. In addition, PCPs reported that they are not confident that they will be able to obtain timely consultations from specialty mental health providers. Only about $50 \%$ of providers stated that they are able to receive consultation on a complicated case or assistance with a psychiatric emergency. ${ }^{10}$ This lack of confidence on various levels can lead to suboptimal care or a barrier to providing any mental health treatment at all. Furthermore, there is a heightened awareness of patient confidentiality issues surrounding mental health disorders, which may further discourage providers from becoming involved in behavioral health care.

\section{Potential Interventions}

In order to assess methods to eliminate some of these barriers and gain confidence to forge ahead, we need to look at what has been already studied. By reviewing different educational and organizational interventions implemented to improve the care for depression, some conclusions can be made. ${ }^{11}$ It appears that well- 
FIGURE 2 Chronic Care Model

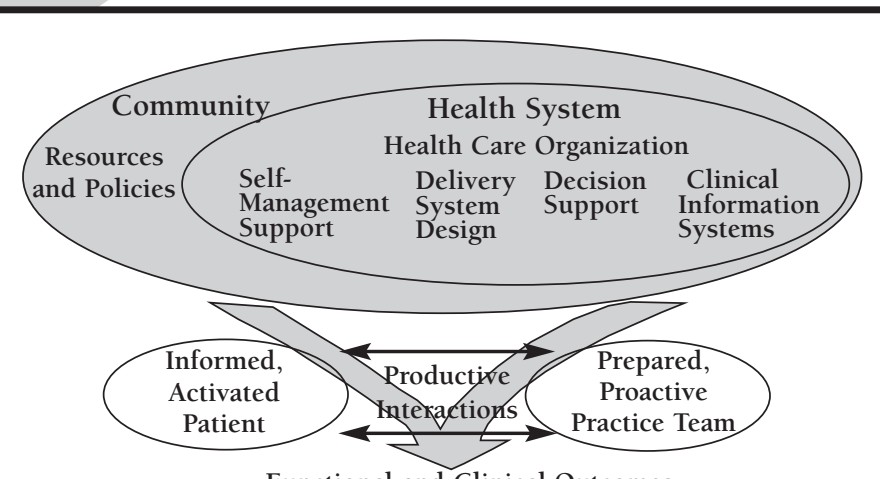

Functional and Clinical Outcomes

The chronic care model links the community and health care system by encompassing 6 components-resources and policies, health care organization, self-management support, delivery system design, decision support, and clinical information systemsto produce productive interactions between informed activated patients and a prepared proactive practice team, which, in turn, results in good outcomes.

developed clinician education (e.g., videotapes, small group teaching, role play) does not work if there is no organizational support. Obtaining feedback on medication adherence and guideline decisions without organizational support also is not effective. Group health reported that there was no change in outcomes when PCPs were simply provided with information about premature discontinuation of antidepressant treatment by their patients. ${ }^{12}$ If they were not provided a mechanism for contacting or following up with the patients, there was little benefit. Interventions that were short-term (i.e., lasting less than one year) also were not effective. ${ }^{11}$

On the other hand, there are strategies that are effective, such as complex interventions that incorporate clinician education, defined roles for care managers, and interaction with mental health specialists. ${ }^{11}$ Medication counseling via telephon ${ }^{13}$ is also effective. Utilizing clinical pharmacists in the care manager role ${ }^{14}$ has also worked successfully. Pharmacists have the knowledge and expertise to educate patients about medications and the importance of adherence. Although chronic care models exist for other diseases, there is much evidence for their effectiveness in the area of depression. ${ }^{15}$ The chronic care model encompasses both the community and the health system (Figure 2) ${ }^{16}$ The byproducts of this model are informed, activated patients and prepared, proactive practice teams to obtain improved outcomes.

\section{Organization of the Health Care System}

In order for the chronic care model to succeed, the organization of the health care system needs to be examined. There must be a culture, organization, and mechanism that promotes the delivery of safe, high-quality care. The leadership of the health care system needs to recognize the importance of the model even when resources are scarce. An organization that focuses only on shortterm goals and the financial bottom line does not do nearly as well as an organization that accepts a culture based on high quality over economic gain. ${ }^{17}$

PCPs need good decision-support systems to succeed in a chronic care model. The accessibility and usefulness of evidencebased guidelines are crucial. Surveys have demonstrated that only half of the physicians stated that guidelines were helpful, ${ }^{18}$ and this is disconcerting. A potential problem with published guidelines provided by organizations such as the American Psychiatric Association (APA) or the Agency for Healthcare Research and Quality (AHRQ), is that only general guidelines on diagnosis and follow-up plans are outlined. There is little detail about strategies for changing medications or utilizing multiple medications. These guidelines often require customization to suit the needs of PCPs. Mental health specialists must be more involved in developing these types of documents. Collaboration can be especially challenging for rural primary care programs that need input about the organization of programs and development of protocols.

Clinical information systems are also essential so that patients can be tracked over time, such as in a depression registry. The type of treatment can be recorded and, in some instances, the system can generate reminders for providers. If there is a depression care manager, his or her notes can be linked to this registry, and these documents can be useful in monitoring performance. While it may seem initially burdensome, this type of information is extremely important to the success of long-term health-quality improvement programs.

In addition to decision support and clinical information systems, there needs to be a delivery system that supports depression care managers. The most important function of the care manager is active follow up in which patients who do not return for a clinic visit can be identified and contacted. The care manager identifies the barrier, if any, and encourages that patient to return to the clinic. It should be stressed that this care manager can be any professional, such as advanced practice nurses, social workers, or clinical pharmacists. The key is to define the competencies and identify the training that care managers will need to competently manage their tasks. Training is also extremely important; too often, care managers complain that they are not very satisfied with the training they have received to competently manage their patients.

Even with the entire organizational and delivery system put in place, the ultimate key player in this model is the patient. Patients struggle daily to identify and complete the next step, whether that step is taking their medication or finding employment. Patients must be encouraged to set a self-management goal and be able to assess their level of confidence in achieving this goal. If their confidence in achieving the goal is less than $70 \%$, another goal should be selected. Due to the chronic nature of the depressive illness, patients must learn how to manage new symptoms and know when to seek help if symptoms return. 
Finally, PCPs should recognize that they cannot work in a silo. They need to develop links to support systems in the community to help them deal with issues such as domestic violence, employment, or housing. Patients should be informed of the various resources in the community that may help them in their recovery from depression. In order for them to be able to develop a more comprehensive treatment plan for patients, providers themselves need to be become more knowledgeable in these areas. Periodic meetings with community groups may be helpful to identify available services and allow coordination among these programs, providers, and patients.

In summary, there is a useful model that can transform the relationship between a PCP and patient so that each encounter is a productive one. Once PCPs are provided with the decision and organizational support to help them manage their patients with depression, they will have increased confidence in treating these patients. Regardless of the outcome, providers will have acquired more knowledge about successful treatment plans, whether that may be as a referral or continuation of treatment in their office. Success of this model is contingent upon the support of the entire health care system.

\section{DISCLOSURES}

This article is based on the proceedings of a symposium held on October 6 , 2005, at the Academy of Managed Care Pharmacy's 2005 Educational Conference in Nashville, Tennessee, which was sponsored by an educational grant from Wyeth. Dr. Ford received an honorarium from Wyeth for participation in the symposium. While he presented this information, including the table and figures, at the symposium, he was not involved in the preparation and approval of this written account of his presentation. Ford discloses no potential bias or conflict of interest relating to this article.

\section{REFERENCES}

1. Schreuders B, van Oppen P, van Marwijk HWJ, Smit JH, Stalman WAB. Frequent attenders in general practice: problem solving treatment provided by nurses. BMC Fam Pract. 2005;6:42.

2. Smith MD, McGhan WF. The high cost of anxiety—anxiety disorders. Bus Health. August 1997. Available at:

http://www.findarticles.com/p/articles/mi_m0903/is_n8_v15/ai_20420588. Accessed on March 3, 2006.

3. Stafford RS, MacDonald EA, Finkelstein SN. National patterns of medication treatment for depression, 1987 to 2001. Prim Care Companion J Clin Psychiatry. 2001;3(6):232-35.
4. Aikens JE, Nease DE, Nau DP, Klinkman MS, Schwenk TL. Adherence to maintenance-phase antidepressant medication as a function of patient beliefs about medication. Ann Fam Med. 2005;3(1):23-30.

5. Williams JW, Rost K, Dietrich AJ, Ciotti MC, Zyzanski SJ, Cornell J. Primary care physicians' approach to depressive disorders effects of physician specialty and practice structure. Arch Fam Med. 1999;8:58-67.

6. Patient Health Questionnaire-9 (PHQ-9). Available at:

http://www.pfizer.com/pfizer/phq-9/index.jsp. Accessed February 26, 2006.

7. Van Voorhees BW, Cooper LA, Rost KM, et al. Primary care patients with depression are less accepting of treatment than those seen by mental health specialists. J Gen Intern Med. 2003;18(12):991-1000.

8. Rubenstein LV, Swanson KA, Meredith L, Lee ML, Ford DE, Rost KM. Are discomfort with depression care and/or clinical uncertainty associated with burnout among primary care clinicians? Presented at: 26th Annual Meeting of the Society of General Internal Medicine; April 30-May 3, 2003; Vancouver, British Columbia, Canada. J Gen Intern Med. 2003;18(suppl 1): 254.

9. Van Voorhees BW, Wang N-Y, Ford DE. Managed care organizational complexity and access to high-quality mental health services: perspective of US primary care physicians. Gen Hosp Psychiatry. 2003;25:149-57.

10. Trude S, Stoddard JJ. Primary Care Physicians and Mental Health Services. J Gen Intern Med. 2003;18:442.

11. Gilbody S, Whitty P, Grimshaw J, Thomas R. Educational and organizational interventions to improve the management of depression in primary care. A systematic review. JAMA. 2003;389:3145-51.

12. Thompson C, Kinmonth AL, Stevens L, et al. Effects of a clinical-practice guideline and practice-based education on detection and outcome of depression in primary care: Hampshire Depression Project randomised controlled trial. Lancet. 2000;355(9199):185-91.

13. Simon G, VonKorff M, Rutter C, Wagner E. A randomized trial of monitoring, feedback, and management of care by telephone to improve depression treatment in primary care. BMJ. 2000;320:550-54.

14. Bero LA, Mays NB, Barjesteh K, Bond C. Expanding the roles of outpatient pharmacists: effects on health services utilisation, costs, and patient outcomes [abstract of review]. Cochrane Database Syst Rev. 2000;(2):CD000336.

15. Tsai AC, Morton SC, Mangione CM, Keeler EB. A meta-analysis of interventions to improve care for chronic illnesses. Am J Manag Care. 2005;11(8): $478-88$.

16. Wagner EH. Chronic disease management: what will it take to improve care for chronic illness? Eff Clin Pract. 1998;1:2-4.

17. Cretin S, Shortell SM, Keeler E. An Evaluation of Collaborative Interventions to Improve Chronic Illness Care. Eval Rev. 2004;38:28-51.

18. Katon W, Van Korff M, Lin E, et al: Collaborative management to achieve treatment guidelines: impact on depression in primary care. JAMA. 1995;273: 1026-31. 